\title{
APLICAÇÃO DA ANÁLISE DE REGRESSÃO LINEAR NA PREDIÇÃO DO COEFICIENTE DE EXPANSÃO TÉRMICA DOS LÍQUIDOS IÔNICOS IMIDAZÓLICOS [HMIM][PF6], [BMIM][TF2N] E [BMIM][PF6]
}

\author{
APPLICATION OF LINEAR REGRESSION ANALYSIS OF THE PREDICTION OF THE \\ THERMAL EXPANSION COEFFICIENT OF IMIDAZOLE IONIC LIQUIDS \\ [HMIM][PF6], [BMIM][TF2N] AND [BMIM][PF6]
}

\author{
César Augusto Canciam \\ Universidade Tecnológica Federal do Paraná - Campus Ponta Grossa, Avenida Monteiro Lobato, km 04, \\ Ponta Grossa, Paraná, CEP: 84016-210, canciam@utfpr.edu.br
}

\begin{abstract}
RESUMO
Os líquidos iônicos são compostos com estrutura iônico-covalente que apresentam pontos de fusão abaixo de $100^{\circ} \mathrm{C}$, baixas pressões de vapor, não são inflamáveis e são relativamente atóxicos. Devido a essas características, os líquidos iônicos têm despertado grande interesse principalmente com relação à potencialidade de substituir os solventes orgânicos convencionais nos mais diversos processos. O presente trabalho teve por objetivo predizer os valores para o coeficiente de expansão térmica dos líquidos iônicos imidazólicos: hexafluorofosfato de 1-metil-3-hexilimidazólio ([HMIM][PF 6$]$ ), bis(trifluorosulfonilo)imideto de 1-butil-3-metilimidazólio ([BMIM] $\left[\mathrm{Tf}_{2} \mathrm{~N}\right]$ ) e hexafluorofosfato de 1-butil-3-metilimidazólio ([BMIM][PF 6$]$ ). Para tanto, através da análise de regressão linear da densidade de cada um dos líquidos iônicos em função da temperatura foi possível predizer os valores para o coeficiente de expansão térmica. Estes dados experimentais foram obtidos de outros trabalhos. Os valores encontrados para o coeficiente de expansão térmica são $6,15 \mathrm{x}$

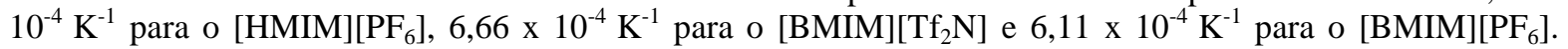
Embora não terem sido encontrados valores experimentais para os líquidos iônicos estudados, os valores encontrados neste trabalho apresentam-se próximos a outro líquido iônico imidazólico.
\end{abstract}

Palavras-chave: Dilatação; Coeficiente; Líquidos iônicos; Predição; Regressão.

\begin{abstract}
Ionic liquids are substances of ionic-covalent structure which have melting points below $100^{\circ} \mathrm{C}$, low vapor pressures, are not flammable and relatively nontoxic. Due to these characteristics, ionic liquids have showed potencial to replace conventional organic solvents in various processes. This work aimed to predict values for the thermal expansion coefficient of the following imidazole ionic liquids: 1-methyl-3-hexylimidazolium hexafluorophosphate ([HMIM][PF6]), 1-butyl-3-methylimidazolium bis(trifluorosulfonyl)imide ([BMIM][Tf2N]) and 1-butyl-3-methylimidazolium hexafluorophosphate ([BMIM][PF6]). Through the linear regression analysis of each of ionic liquids with regard to the density as a temperature function, was possible to predict the values for the thermal expansion coefficient. The experimental data used were obtained from the literature. The values found for the thermal expansion coefficient are 6.15 x 10-4 K-1 for [HMIM][PF6], 6.66 x 10-4 K-1 for [BMIM][Tf2N] and 6.11 x 10-4 K-1 for [BMIM][PF6]. The obtained values were close to the value available in the literature for another imidazole ionic liquid.
\end{abstract}

Key-words: Thermal Expansion Coefficient; Ionic Liquids; Linear Regression.

\section{INTRODUÇÃO}

Os líquidos iônicos pertencem à classe dos sais orgânicos e são constituídos por cátions orgânicos relativamente grandes e ânions orgânicos ou inorgânicos menores. Esta assimetria na sua composição química reduz a energia reticular do sal, impedindo a formação de uma estrutura cristalina ordenada. Por este motivo, ocorre nos líquidos iônicos uma redução do ponto de fusão. Este ponto de fusão pode estar à temperatura ambiente ou próxima. Em alguns casos, os pontos de fusão estão além de $400^{\circ} \mathrm{C}$ (Valente, 2008). 
De forma geral, os líquidos iônicos apresentam uma energia livre de Gibbs de solvatação negativa (em consequência dos grandes cátions orgânicos e ânions pouco coordenantes e/ou bastante flexíveis), resultando em uma baixa energia reticular e grande entropia (implicando em baixo ponto de fusão). Ou seja, o estado líquido é termodinamicamente favorável para estes compostos, mesmo em baixas temperaturas (Rodrigues, 2010).

A ausência de uma estrutura cristalina ordenada confere aos líquidos iônicos: elevada capacidade calorífica, volatitilidade extremamente baixa, baixas inflamabilidade e toxicidade; estabilidade térmica e condutividade iônica elevadas, ampla gama de temperatura no estado líquido, densidades superiores à da água, viscosidades comparadas a óleos, miscibilidade em uma larga gama de concentrações em substâncias polares e apolares (em decorrência da elevada capacidade de solvatação) e capacidade de absorver moléculas menores $\left(\mathrm{CO}_{2}, \mathrm{SO}_{2}, \mathrm{CS}_{2}\right.$, entre outras) (Valente, 2008; Ando; Santos, 2011).

Devido à reduzida volatilidade (evitando a emissão de compostos orgânicos) e baixa toxicidade, os líquidos iônicos são ecologicamente aceitáveis e considerados "amigos" do ambiente (Silva et al., 2005; Valente, 2008). Lima (2011) classificou os líquidos iônicos como "meios alternativos ambientalmente seguros", pois oferecem poucos riscos ao ambiente durante o processo, além de possibilitar a separação dos produtos e a recuperação do solvente com baixo consumo de energia.

Consorti e colaboradores (2001) comentaram da utilização dos líquidos iônicos como solventes (em reações orgânicas, em catálise bifásica, na extração líquido-líquido e na análise espectroscópica de compostos metálicos) e como fase estacionária para cromatografia gasosa.

Utilizam-se líquidos iônicos como tensoativos, como catalisadores ácidos nas reações orgânicas e cossolventes em reações enzimáticas. Em eletroquímica, os líquidos iônicos são eletrólitos em diversas tecnologias, por exemplo, em baterias secundárias, células fotoelétricas e de combustíveis, capacitores elétricos de dupla camada e outros dispositivos eletroquímicos (Consorti et al., 2001; Valente, 2008).

Os líquidos iônicos imidazólicos apresentam-se como estruturas supramoleculares poliméricas, altamente organizadas, em que cada cátion imidazólio é circundado de ânions e cada ânion é circundado de cátions imidazólios, através das ligações de hidrogênio existentes entre os hidrogênios do ciclo imidazólico e os ânions (Migliorini, 2009).

Com relação aos líquidos iônicos imidazólicos, existe um grande interesse na sua utilização, pois apresentam: estabilidade ao ar e umidade (não sofrem decomposição na presença de ar e água), baixa inflamabilidade, alta estabilidade térmica, pressão de vapor muito baixa, amplas janelas eletroquímicas, altas condutividades (elétrica e térmica), altas mobilidades iônicas e fácil recuperação e reutilização (Dupont, 2004; Migliorini, 2009).

De acordo com Rogers e colaboradores (2002), os líquidos iônicos imidazólicos [HMIM] $\mathrm{PF}_{6}$ ] $\left(\mathrm{C}_{10} \mathrm{H}_{19} \mathrm{~N}_{2} \mathrm{PF}_{6}\right)$, [BMIM] $\left.\mathrm{Tf}_{2} \mathrm{~N}\right]\left(\mathrm{C}_{10} \mathrm{H}_{15} \mathrm{~N}_{3} \mathrm{O}_{4} \mathrm{~S}_{2} \mathrm{~F}_{6}\right)$ e [BMIM] [ $\left.\mathrm{PF}_{6}\right]\left(\mathrm{C}_{8} \mathrm{H}_{15} \mathrm{~N}_{2} \mathrm{PF}_{6}\right)$ apresentam-se como líquidos viscosos, de coloração que vai de incolor a levemente amarelada. Todos são insolúveis em água e sem odor característico. A Tabela 1 relaciona o ponto de fusão dessas substâncias.

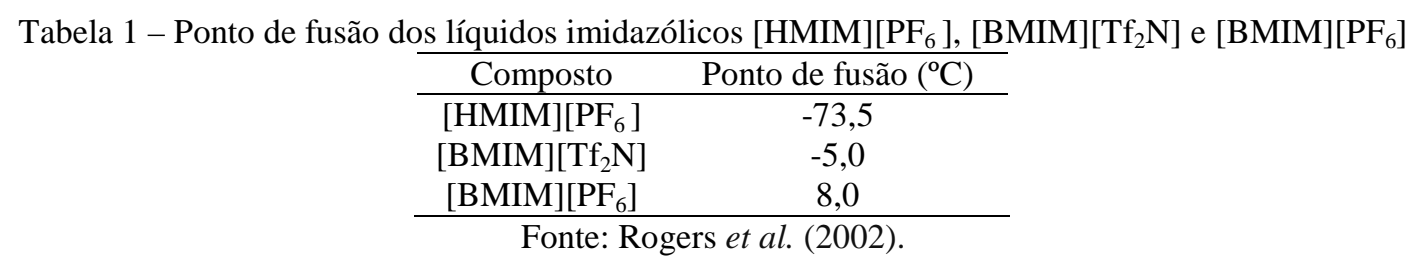

Segundo Chung e colaboradores (1996), a densidade é uma propriedade da matéria intimamente relacionada com a temperatura e a pressão. A densidade é uma das mais fundamentais propriedades de um material e está intrinsicamente relacionada com outras propriedades termofísicas. Uma dessas propriedades é o coeficiente de expansão térmica $(\beta)$. 
O coeficiente de expansão térmica está relacionado com a resposta dos materiais à aplicação de calor (Callister, 2008). Sendo uma propriedade termodinâmica que fornece uma medida da variação da densidade em resposta a uma mudança na temperatura, sob uma condição de pressão constante (Incropera; Dewitt, 1998).

A equação (1) relaciona a densidade com a variação da temperatura e o coeficiente de expansão térmica, em que $T_{0}$ e $T$ correspondem, respectivamente, à temperatura inicial e à temperatura final; e, $d_{0} \mathrm{e} d$ correspondem, respectivamente, às densidades nessas temperaturas (Canciam, 2005).

$$
\ln \left(\frac{d_{0}}{d}\right)=\beta \cdot\left(T-T_{0}\right)
$$

Assim, com base nos dados da densidade em função da temperatura, consegue-se determinar o coeficiente de expansão térmica $(\beta)$ (Santos; Vieira, 2010).

De acordo com Canciam (2010), a equação (1) corresponde a uma função afim. Dessa forma, o gráfico de $\ln \left(\frac{d_{0}}{d}\right)$ versus $\left(T-T_{0}\right)$ fornece uma reta, em que o coeficiente angular corresponde, numericamente, ao coeficiente de expansão térmica $(\beta)$.

A partir do conhecimento do coeficiente de expansão térmica $(\beta)$ é possível avaliar os impactos no sistema de medição volumétrico devido ao aumento da temperatura (Santos; Vieira, 2010).

O objetivo deste trabalho foi predizer os valores do coeficiente de expansão térmica $(\beta)$ dos líquidos iônicos imidazólicos: hexafluorofosfato de 1-metil-3-hexilimidazólio ([HMIM][PF $]$ ), bis(trifluorosulfonilo)imideto de 1-butil-3-metilimidazólio ([BMIM][Tf $\left.{ }_{2} \mathrm{~N}\right]$ ) e hexafluorofosfato de 1butil-3-metilimidazólio ([BMIM] $\left.\left[\mathrm{PF}_{6}\right]\right)$. A predição dos coeficientes de expansão térmica utilizou dados da densidade em função da temperatura encontrados em outros trabalhos.

\section{MATERIAL E MÉTODOS}

A Tabela. 2 indica os dados da densidade dos líquidos iônicos imidazólicos [HMIM][PF 6 e [BMIM] $\left[\mathrm{Tf}_{2} \mathrm{~N}\right]$ (à pressão atmosférica) em função da temperatura. Estes dados foram adaptados do trabalho de Harris e colaboradores (2007).

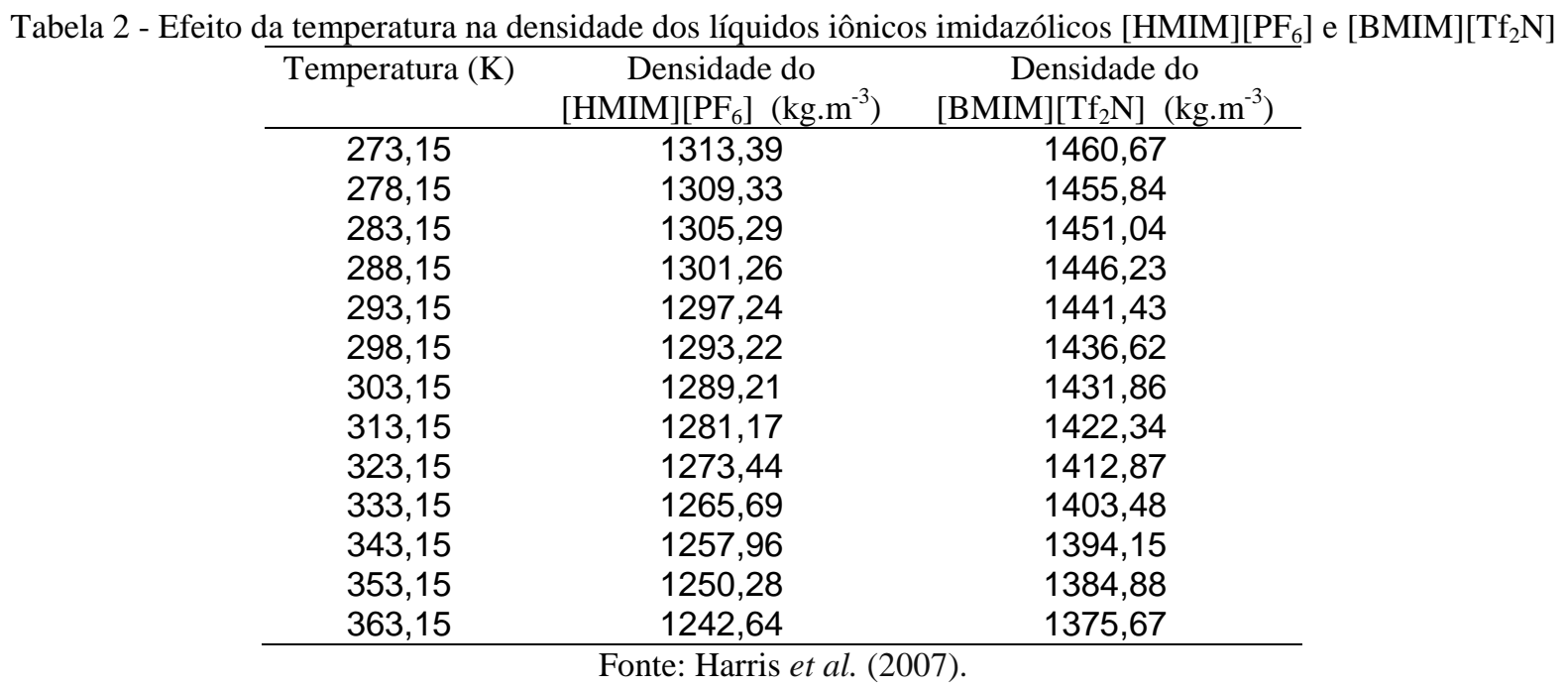


A Tabela 3 indica os dados da densidade do líquido iônico imidazólico [BMIM] $\left[\mathrm{PF}_{6}\right.$ ] (à pressão atmosférica) em função da temperatura. Estes dados foram adaptados do trabalho de Fan $e t$ al.(2009).

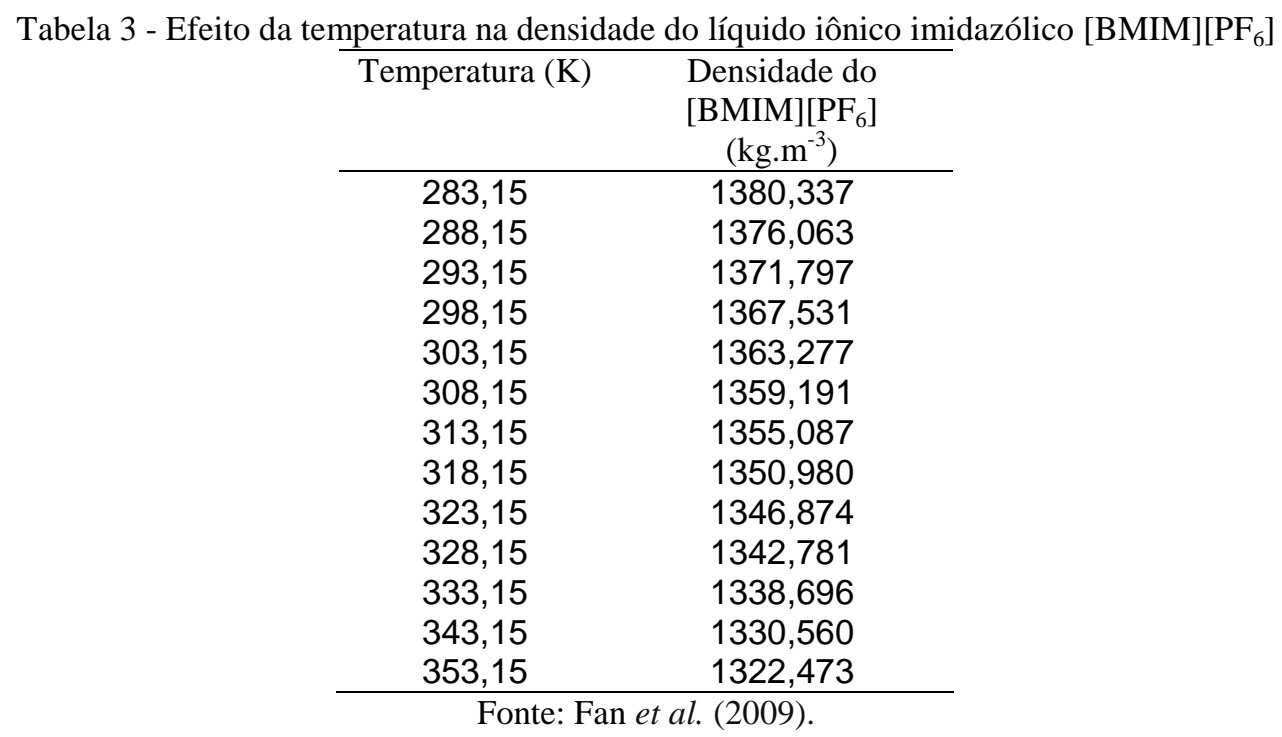

Para a análise de regressão linear foram consideradas as equações (2) a (9). Estas equações são adaptações do trabalho de Triola (2008).

$$
\begin{gathered}
\beta=\left\{\frac{n \cdot a-b}{n \cdot c-h}\right\} \\
R^{2}=\frac{(n \cdot a-b)}{(\sqrt{(n \cdot c-h)} \cdot(\sqrt{(n \cdot e-f)})} \\
a=\left\{\sum\left[\left(T-T_{0}\right) \cdot \ln \left(d_{0} / d\right)\right]\right\} \\
b=\left\{\left[\sum\left(T-T_{0}\right)\right]\left[\sum \ln \left(d_{0} / d\right)\right]\right\} \\
c=\sum\left(T-T_{0}\right)^{2} \\
e=\sum\left[\ln \left(d_{0} / d\right)\right]^{2} \\
f=\left[\sum \ln \left(d_{0} / d\right)\right]^{2} \\
h=\left[\sum\left(T-T_{0}\right)\right]^{2}
\end{gathered}
$$

Sendo $n$ correspondente ao número de dados amostrais emparelhados. Com base nas Tabelas 2 e $3, n$ equivale a 13 .

A equação (2) e a equação (3) determinam, respectivamente, o coeficiente de expansão térmica $(\beta)$ e o coeficiente de correlação $\left(R^{2}\right)$. 
Aplicando os dados das Tabelas 2 e 3 nas equações (2) a 9, foi possível obter o coeficiente de expansão térmica $(\beta)$ e o coeficiente de correlação $\left(R^{2}\right)$ para cada líquido iônico imidazólico.

Nos cálculos, para os líquidos iônicos $[\mathrm{HMIM}]\left[\mathrm{PF}_{6}\right]$ e $[\mathrm{BMIM}]\left[\mathrm{Tf}_{2} \mathrm{~N}\right]$, a temperatura inicial $\left(T_{0}\right)$ corresponde a $273,15 \mathrm{~K}$ e a densidade inicial $\left(d_{0}\right)$, à densidade correspondente a essa temperatura. Para o líquido iônico [BMIM] $\left[\mathrm{PF}_{6}\right]$, a temperatura inicial $\left(T_{0}\right)$ corresponde a $283,15 \mathrm{~K}$ e a densidade inicial $\left(d_{0}\right)$, à densidade correspondente a essa temperatura.

\section{RESULTADOS E DISCUSSÃO}

A Figura. 1 ilustra o gráfico de $\ln \left(\frac{d_{0}}{d}\right)$ versus $\left(T-T_{0}\right)$ para os líquidos iônicos imidazólicos estudados.

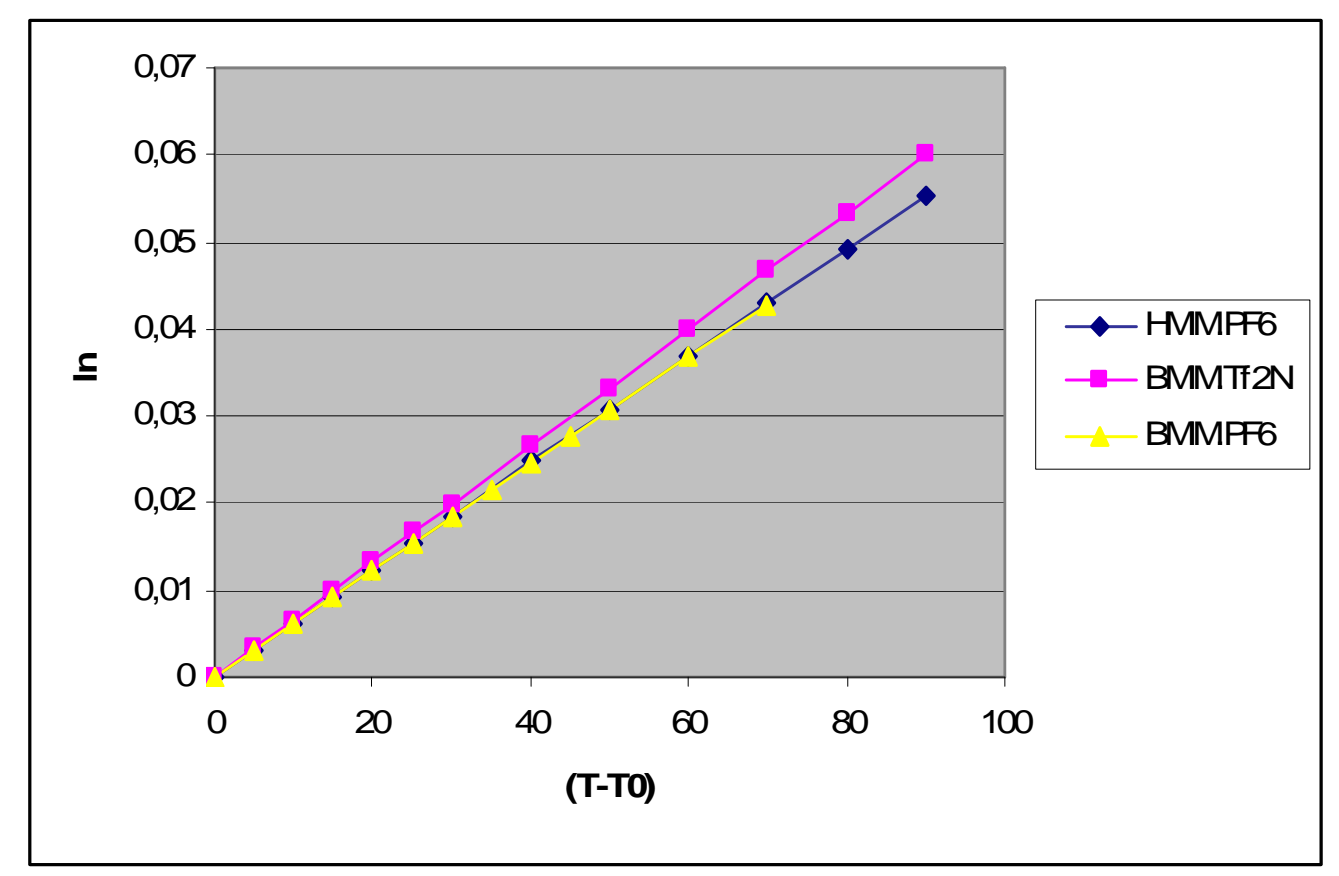

Figura 1 - Gráfico de $\ln \left(\frac{d_{0}}{d}\right)$ versus $\left(T-T_{0}\right)$ para os líquidos iônicos imidazólicos estudados

A Tabela 4 relaciona os valores encontrados para o coeficiente de expansão térmica $(\beta)$ através da equação (2) e para o coeficiente de correlação $\left(R^{2}\right)$ através da equação (3).

Tabela 4 - Valores obtidos para o coeficiente de expansão térmica e para o coeficiente de correlação dos líquidos iônicos imidazólicos estudados

\begin{tabular}{ccc}
\hline Composto & $\beta \cdot 10^{-4}\left(\mathrm{~K}^{-1}\right)$ & $R^{2}$ \\
\hline$[\mathrm{HMIM}]\left[\mathrm{PF}_{6}\right]$ & 6,15 & 0,99 \\
{$[\mathrm{BMIM}]\left[\mathrm{Tf}_{2} \mathrm{~N}\right]$} & 6,66 & 0,99 \\
{$[\mathrm{BMIM}]\left[\mathrm{PF}_{6}\right]$} & 6,11 & 0,99 \\
\hline
\end{tabular}

De acordo com Triola (2008), o coeficiente de correlação $\left(R^{2}\right)$ mede o grau de relacionamento linear entre os valores emparelhados das variáveis dependente e independente em uma amostra, 
avaliando assim, a "qualidade" do ajuste. Ou seja, quanto mais próximo for da unidade, melhor é a "qualidade" do ajuste da função aos pontos do diagrama de dispersão.

Callegari-Jaques (2003), citado por Lira (2004), comentou que para valores do coeficiente de correlação maiores ou iguais a 0,90 e menores que 1,0 a correlação linear é classificada como muito forte. Dessa forma, para todos os compostos indicados na Tabela 4, a correlação linear é classificada como muito forte.

Os valores do coeficiente de expansão térmica $(\beta)$ estão relacionados com a energia de ligação química entre as partículas. Santos e Vieira (2010) comentaram que entre o coeficiente de expansão térmica $(\beta)$ e a energia de ligação química existe uma boa correlação, de maneira que materiais em que as ligações químicas são fortes apresentam baixos coeficientes de expansão térmica.

Os líquidos iônicos, em comparação com os líquidos moleculares convencionais, possuem estrutura mais organizada, apresentando regiões com características bastante distintas. São compostos com regiões polares e apolares, onde ocorrem interações intermoleculares dos tipos eletrostáticas, de hidrogênio e de van der Waals. Como exemplo, enquanto os líquidos moleculares convencionais possuem um arranjo estrutural somente a curta distância (primeiros vizinhos) e por um curto espaço de tempo; nos sólidos cristalinos, a organização estrutural pode ser vista a longas distâncias e por grandes períodos de tempo. Neste sentido, os líquidos iônicos representam um meio termo, pois apresentam arranjo estrutural a médias distâncias e por tempos relativamente longos para um líquido (Rodrigues, 2010). $(\beta)$.

A Tabela 5 relaciona algumas substâncias e seus respectivos coeficientes de expansão térmica

Tabela 5 - Valores do coeficiente de expansão térmica de algumas substâncias

\begin{tabular}{ccc}
\hline Substância & $\beta \cdot 10^{-4}\left(\mathrm{~K}^{-1}\right)$ & Fonte \\
\hline Glicerina & 5,0 & Adaptado de Young; Freedman (2008) \\
Azeite de oliva & 7,20 & Adaptado de Young; Freedman (2008) \\
Óleo de gergelim & 7,46 & Adaptado de Canciam (2010) \\
\hline
\end{tabular}

Comparando os valores indicados nas Tabelas 4 e 5, pode-se observar que os coeficientes de expansão térmica para os líquidos iônicos imidazólicos estudados encontram-se entre os coeficientes de expansão térmica da glicerina e do azeite de oliva.

Segundo Castelló (2009), a glicerina corresponde a um poliálcool, com um grupamento hidroxila em cada um de seus três átomos de carbono. Isto confere à glicerina a facilidade em formar ligações de hidrogênio entre suas moléculas. Estas interações intermoleculares são significativamente fortes.

O azeite de oliva e o óleo de gergelim, assim como todos os óleos vegetais, ocorrem interações do tipo van der Waals entre suas moléculas. Em comparação com as ligações de hidrogênio, as interações do tipo van der Waals são mais fracas (CANCIAM, 2010).

De acordo com Brown e Holme (2009), as forças de van der Waals são originadas a partir da rápida flutuação da densidade eletrônica de cada átomo, que induz a formação de um momento elétrico entre os átomos vizinhos, fazendo com que estes se atraiam.

Dessa forma, sugere-se que os líquidos iônicos imidazólicos estudados, em termos do coeficiente de expansão térmica, representam um meio termo entre os líquidos com interações intermoleculares do tipo ligações de hidrogênio e líquidos com interações intermoleculares do tipo van der Waals.

A Tabela 6 associa as massas molares $(\mathrm{MM})$ e os coeficientes de expansão térmica $(\beta)$ para os líquidos iônicos imidazólicos estudados.

A Figura 2 ilustra o gráfico do coeficiente de expansão térmica $(\beta)$ versus massa molar (MM). 
Observando a Figura 2, sugere-se que existe uma correlação entre o coeficiente de expansão térmica e a massa molar, de maneira que um aumento da massa molar do líquido iônico imidazólico implica em um aumento do coeficiente de expansão térmica.

Tabela 6 - Associação entre os valores do coeficiente de expansão térmica e a massa molar dos líquidos iônicos imidazólicos estudados

\begin{tabular}{ccc}
\hline Composto & $\beta \cdot 10^{-4}\left(\mathrm{~K}^{-1}\right)$ & $\begin{array}{c}\mathrm{MM} \\
\left(\mathrm{g} \cdot \mathrm{mol}^{-1}\right)\end{array}$ \\
\hline$[\mathrm{BMIM}]\left[\mathrm{PF}_{6}\right]$ & 6,11 & 284,18 \\
{$[\mathrm{HMIM}]\left[\mathrm{PF}_{6}\right]$} & 6,15 & 312,24 \\
{$[\mathrm{BMIM}]\left[\mathrm{Tf}_{2} \mathrm{~N}\right]$} & 6,66 & 419,36 \\
\hline
\end{tabular}

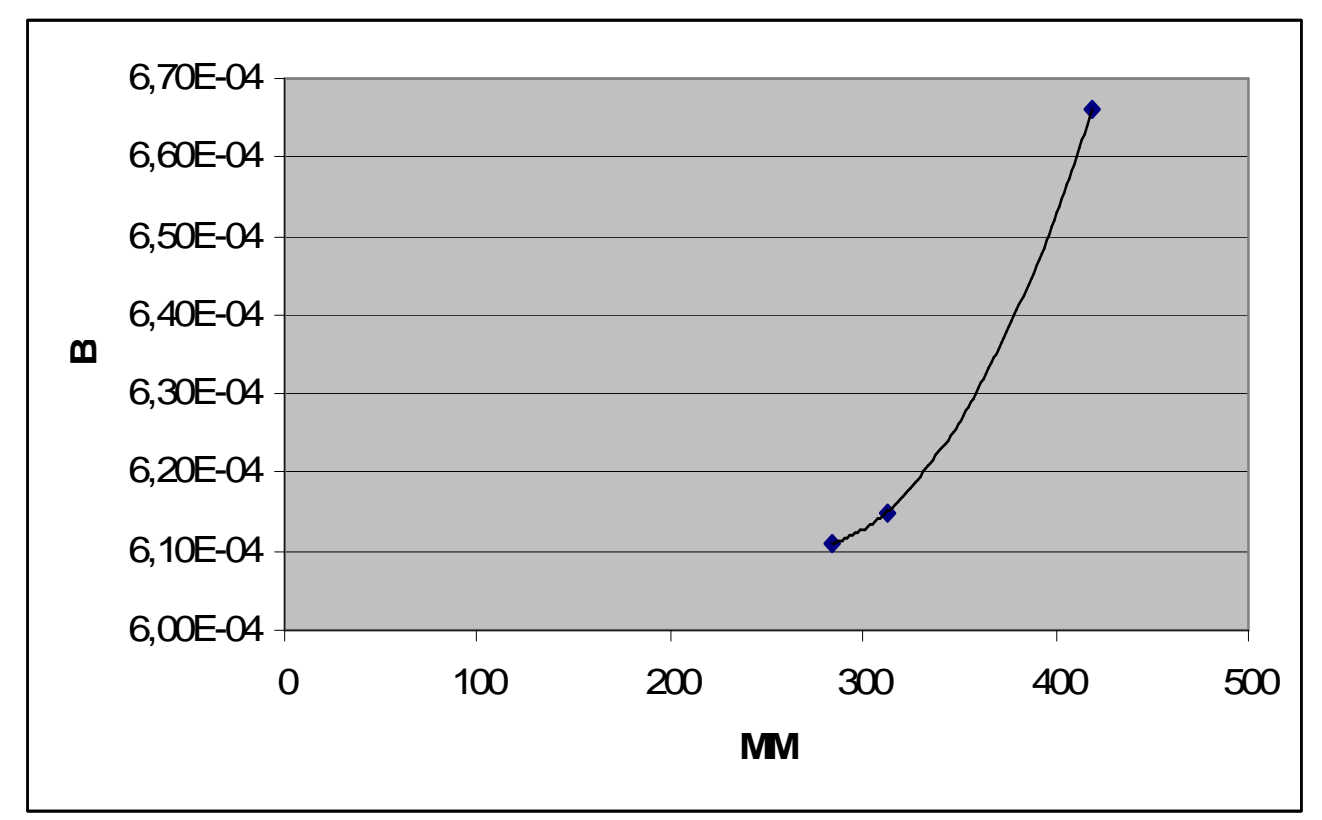

Figura 2 - Gráfico do coeficiente de expansão térmica $(\beta)$ versus a massa molar (MM) para os líquidos iônicos imidazólicos estudados

Com relação aos impactos da dilatação térmica sobre o sistema de medição volumétrica, a Tabela 7 indica a ordem de aumento de volume para uma variação de temperatura de 10K, considerando um volume de 1000 litros para cada líquido iônico imidazólico.

Tabela 7 - Ordem de aumento do volume

\begin{tabular}{cc}
\hline Composto & Ordem de aumento \\
& $(\mathrm{L})$ \\
\hline$[\mathrm{BMIM}]\left[\mathrm{PF}_{6}\right]$ & 6,11 \\
{$[\mathrm{HMIM}]\left[\mathrm{PF}_{6}\right]$} & 6,15 \\
{$[\mathrm{BMIM}]\left[\mathrm{Tf}_{2} \mathrm{~N}\right]$} & 6,66 \\
\hline
\end{tabular}

$\mathrm{Na}$ literatura não foram encontrados valores experimentais para os coeficientes de expansão

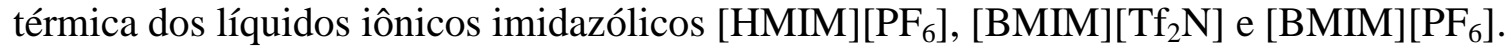

Lu e colaboradores (2005) estudando algumas propriedades do tetracloreto de gálio e 1-metil3-butilimidazólio ([BMIM] $] \mathrm{GaCl}_{4}$ ), encontraram, experimentalmente para este líquido iônico imidazólico, um coeficiente de expansão térmica igual a $6,00 \times 10^{-4} \mathrm{~K}^{-1}$. Com isso, pode-se constatar que os valores encontrados neste trabalho estão próximos a outro líquido iônico imidazólico. 


\section{CONCLUSÃO}

O coeficiente de expansão térmica $(\beta)$ para os líquidos iônicos imidazólicos estudados variou de $6,11 \times 10^{-4} \mathrm{~K}^{-1}$ do hexafluorofosfato de 1-butil-3-metilimidazólio ([BMIM][PF 6$]$ ) a $6,66 \times 10^{-4} \mathrm{~K}^{-1}$ do bis(trifluorosulfonilo)imideto de 1-butil-3-metilimidazólio ([BMIM][Tf $2 \mathrm{~N}]$ ). Estes valores estão próximos a outro líquido iônico imidazólico.

Em todos os casos, o coeficiente de correlação $\left(R^{2}\right)$ foi de 0,99 , classificando todas as correlações lineares como muito fortes.

Em termos dos valores dos coeficientes de expansão térmica, os líquidos iônicos imidazólicos estudados estão entre o azeite de oliva (interações intermoleculares do tipo van der Waals) e a glicerina (interações intermoleculares do tipo ligações de hidrogênio).

Os resultados sugerem que existe uma correlação entre o coeficiente de expansão térmica e a massa molar do líquido iônico imidazólico, de maneira que um aumento da massa molar do líquido iônico imidazólico implica em um aumento do coeficiente de expansão térmica.

Sobre os impactos da dilatação térmica no sistema de medição volumétrico, a ordem de aumento no volume dos líquidos iônicos imidazólicos estudados variou de 6,11 litros (hexafluorofosfato de 1-butil-3-metilimidazólio) ([BMIM][PF $]$ ) a 6,66 litros (bis(trifluorosulfonilo)imideto de 1-butil-3-metilimidazólio) ([BMIM][Tf $\left.\left.{ }_{2} \mathrm{~N}\right]\right)$, considerando uma variação de 10K para cada 1000 litros de líquido iônico imidazólico.

\section{REFERÊNCIAS}

ANDO, R.A.; SANTOS, P.S. Interação de $\mathrm{SO}_{2} \mathrm{CS}_{2}$ com líquidos iônicos imidazólicos. In: REUNIÃO ANUAL DA SOCIEDADE BRASILEIRA DE QUÍMICA, 34a , Florianópolis. Anais. Sociedade Brasileira de Química, 2011.

BROWN, L. S.; HOLME, T. A. Química geral aplicada à Engenharia, São Paulo: Cengage Learning, 2009. 656p.

CALLISTER, W.D. Ciência e Engenharia de Materiais: uma introdução. 7.ed. Rio de Janeiro : LTC, 2008. 590p.

CANCIAM, C.A. Predição do coeficiente de expansão térmica do óleo de algodão. Publicatio UEPGCiências Exatas e da Terra, Ciências Agrárias e Engenharias, v. 11, n. 3, p.27-31, dez. 2005.

CANCIAM, C.A. Predição do coeficiente de expansão térmica do óleo de gergelim (Sesamum indicum L.) através da aplicação de regressão linear. E-xacta, v. 3, n. 1, p.20-28, 2010.

CASTELLÓ, M.L. Estudo da glicerina por análises térmicas. 2009. 99f. Dissertação (Mestrado em Ciências) - Universidade Federal do Rio de Janeiro, Rio de Janeiro.

CONSORTI, T.S.; SOUZA, R.F.; DUPONT, J. Líquidos iônicos contendo o cátion dialquilimidazólio: estrutura, propriedades físico-químicas e comportamento em solução. Química Nova, São Paulo, v. 24, n.6, p. 830-837, març. 2001.

CHUNG, S.K.; THIESSEN, D.B.; RHIM, D.B. A non contact measurement technique for the density and the thermal expansion coefficient of solids and liquid material. Review of Scientific Instruments, v. 67, n. 9, p.3003-3381, 1996. 
DUPONT, J. On the solid, liquid and solution structural organization of imidazolium ionic liquids. Journal of the Brazilian Chemical Society, v. 15, n. 6, p. 341-350, mai. 2004.

FAN, W.; ZHOU, Q.; SUN, J.; ZHANG, S. Density, excess molar volume and viscosity for the methyl methacrylate + 1-butyl-3-methylimidazolium hexafluorophosphate ionic liquid binary system at atmospheric pressure. Journal of Chemical \& Engineering Data, v.54, p. 2307-2311, mai. 2009.

HARRIS, K.R.; KANAKUBO, M.; WOOLF, L. A. Temperature and pressure dependence of the viscosity of the ionic liquids 1-hexyl-3-methylimidazolium hexafluorophosphate and 1-butyl- 3methylimidazolium bis(trifluoromethylsulfonyl)imide. Journal of Chemical \& Engineering Data, v. 52, p. 1080-1085, abr. 2007.

INCROPERA, F.P.; DEWITT, D.P. Fundamentos de Transferência de Calor e de Massa. 6. ed. Rio de Janeiro : LTC, 1998. 644p.

LIMA, P.G. Micro-ondas na síntese de líquidos iônicos. Revista Virtual de Química, v. 3, n. 4, p. 325338, out. 2011.

LIRA, S.A. Análise de correlação: abordagem teórica e de construção dos coeficientes com aplicação. 2004. 196f. Dissertação (Mestrado) - Setor de Ciências Exatas e Tecnologia, Universidade Federal do Paraná, Curitiba.

LU, X.M.; XU, W.G.; ZHANG, Q.G.; GUI, J.S.; YANG, J.Z. Studies on the thermodynamic properties of the ionic liquid $\mathrm{BMIGaCl}_{4}$. In: World Congress on Recovery, Recycling and Reintegration, $7^{\circ}$, Beijing. Anais. Chinese Academy of Sciences, 2005.

MIGLIORINI, M.V. Líquidos iônicos para a preparação de híbridos de sílica e suas aplicações na formação de compósitos poliméricos. 2009. 90f. Dissertação (Mestrado em Química), Universidade Federal do Rio Grande do Sul, Porto Alegre.

ROGERS, R.D.; SEDDON, K.R.; VOLKOV, S. Green Industrial Applications of Ionic Liquids. Kluwer Academic Publishers; 2002. 557 p.

RODRIGUES, F. Espectroscopia Raman de líquidos iônicos imidazólicos: interações interiônicas, organização estrutural e efeitos de micro-ambiente. 2010. 173f. Tese (Doutorado em Química), Universidade de São Paulo, São Paulo.

SANTOS, D.Q.; VIEIRA, D.F. Determinação de coeficiente de expansão térmica do biodiesel e seus impactos no sistema de medição volumétrico. Eclética Química, v. 35, n. 4, p.107-112, 2010.

SILVA, F.M., LACERDA, P.S.B.; JONES JUNIOR, J. Desenvolvimento sustentável e Química Verde. Química Nova, v. 28, n. 1, p. 103-110, set. 2005.

TRIOLA, M.F. Introdução à Estatística. 10. ed. Rio de Janeiro: LTC, 2008. 720p.

VALENTE, M.V. Polimerização de PMMA em microemulsão com LIs tensiactivos. 2008. 96f. Dissertação (Mestrado em Engenharia Química), Universidade de Aveiro, Aveiro.

YOUNG, H.D.; FREEDMAN, R.A. Física III - Termodinâmica e Ondas. São Paulo: Editora Addison Wesley LTDA; 2008. 318p. 